\title{
Evaluación de la efectividad de las micorrizas vesículo-arbusculares nativas sobre el desarrollo vegetativo y la producción de maíz chococito cultivado en suelos de vocación minera, en la cuenca alta del río San Juan, Chocó, Colombia
}

\section{Evaluation of the efficiency of the micorrízas native vesiculo-arbusculares on the vegetative development and the production of maize chococito cultivated in soils of mining vocation, in the high basin of the river San Juan, Chocó, Colombia}

\author{
Viky Yency Vega Hurtado, Ing Agrofor ${ }^{1}$, Moisés Mosquera Blandón, Ing Agron ${ }^{2}$ \\ RESUMEN \\ Con el propósito de evaluar la efectividad de las micorrizas $V$. A. nativas sobre \\ el desarrollo vegetativo y productivo de maíz chococito cultivado en zonas del \\ municipio de Tadó, el Instituto de Investigaciones Ambientales del Pacífico \\ (IIAP) determinó que la inoculación de diferentes dosis (0, 100,150 y $250 \mathrm{~g}$ / \\ plántula) del genero Glomus sp. producida por el IIAP, a plántulas de dicha \\ especie en fase de vivero, produjo una efectiva y eficaz inoculación. Se aplicó \\ el procedimiento del Manual de Métodos para la investigación de las micorrizas \\ V. A. y otros simbiontes en el laboratorio, propuesto por Sieverding (1983) y se \\ desarrollaron cuatro fases en campo. Los resultados demuestran que bajo las \\ condiciones agroambientales de la zona de estudio, la efectiva relación \\ 1. Contratista, Instituto de Investigacio- \\ nes Ambientales del Pacífico (IIAP), \\ Quibdó, Colombia. \\ e-mail: vikyvega15@gmail.com \\ 2. Especialista en Gerencia de Recursos \\ Naturales, Investigador principal del \\ Componente Productivo, Instituto de \\ Investigaciones Ambientales del Pacífi- \\ co (IIAP), Quibdó, Colombia. \\ e-mail: mmosquera@iiap.org.co \\ Recibido: 8 de julio de 2011 \\ Aceptado: 28 de octubre de 2011
} simbiótica establecida entre el inóculo aplicado y planta de maíz chococito, mejoró su desarrollo foliar, desarrollo longitudinal, grosor del tallo, número y tamaño de mazorcas y producción. Los tratamientos 150 y $250 \mathrm{~g} / \mathrm{plán}$ tula generaron la mejor efectividad y mayores diferencias significativas respecto al testigo, pero no entre ellos.

Palabras clave: Simbionte; Inóculo; Inoculación; Micorrizas V. A; Glomus sp. Bioabono; Relación simbiótica.

\begin{abstract}
In order to evaluate the effectiveness of VA mycorrhizal native vegetation on the development and production of maize grown in areas chococito municipality of Tado, Instituto de Investigaciones Ambientales del Pacifico (IIAP) found that the inoculation of different doses $(0,100.150$ and $250 \mathrm{~g} / \mathrm{seedling})$ of the genus Glomus sp. produced by the IIAP to seedlings of this species in nursery stage, produced an effective and efficient inoculation. Procedure was applied Manual of Methods for the Investigation of Mycorrhizae $V$. A. and other symbionts in the Laboratory, proposed by E. Sieverding (1983) and developed four (4) phases in the field. The results show that under agri-environmental conditions of the study area, the effective symbiotic relationship established between the inoculum applied and chococito corn plant, improved leaf development, development length, stem thickness, number and size of ears and production. Treatments 150 and $250 \mathrm{~g} / \mathrm{seedling}$ generated the best ERA and more significant differences compared with the control, but not between them.
\end{abstract}




\section{Bioetnia Volumen 8 No 2 (julio-diciembre), 2011}

Keywords: Symbiont; Inoculum; Inoculation; Mycorrhiza VA; Biofertilizer; Glomus sp.; Symbiotic relationship.

\section{INTRODUCCIÓN}

El maíz (Zea mays), es uno de los agroalimentos de mayor importancia en cuanto a la dieta alimentaria de los pueblos negros e indígenas del Chocó, en particular de las comunidades que habitan en las zonas de la cuenca alta del río San Juan, pero en los últimos años ha visto reducida su frontera de siembra y disminuidos drásticamente sus índices de productividad y niveles de producción por diferentes factores, entre ellos, la pérdida de capacidad productiva de los suelos y en algunos casos la baja vigorosidad y viabilidad de las semillas nativas.

La problemática edáfica se relaciona con las deficiencias y lixiviación de elementos nutrientes, bajo contenido de materia orgánica y alta acidez de estos suelos, lo que de forma directa afecta la fertilidad de los mismos; por consiguiente, limita una eficiente nutrición y desarrollo de las plantas, reduce la producción de mazorcas y en general la cosecha de este importante cereal. La excesiva precipitación (rango entre 8.000 a $12.000 \mathrm{~mm} / \mathrm{año}$ ) genera procesos de lixiviación y alta acidez, que constituyen una limitante para el desarrollo vegetativo y productivo de los cultivos, porque las plantas no encuentran la cantidad necesaria de elementos nutrientes disponibles para su normal crecimiento (Malagón 2002).

Otro factor que incide fuertemente en esta situación es la minería irracional, que en muchos municipios y corregimientos del Chocó es una de las principales actividades económicas; por la minería, se han perdido grandes extensiones de suelo aptas para la agricultura. Muchos municipios del departamento del Chocó, especialmente los municipios pertenecientes a la cuenca alta del río San Juan, son totalmente dependientes en materia agroalimentaria, esto porque además de las condiciones descritas, la mayoría de sus habitantes se dedican a la actividad minera tradicional (barequeo) y/o semi-industrial (retroexcavadora), obligando a los moradores del municipio a abastecer su canasta alimentaria de departamentos vecinos, aumentando así el costo de vida y agudizando la crisis agroalimentaria, como consecuencia de no encontrar zonas óptimas u otras alternativas para establecer especies agrícolas que anteriormente se cultivaban en estos lugares, lo que atenta contra el derecho que tienen las comunidades de tener garantías de seguridad y soberanía alimentaria.

El término micorrizas se refiere a la asociación simbiótica mutualística, que se desarrolla entre las raíces de la mayoría de las plantas superiores y ciertos hongos que son comunes en el suelo (Gianinazzi 1991; Bethlenfalvay, Linderman 1992). En ella, el micelio del hongo infectado se asocia con la corteza radical a modo de endófito y proyecta sus hifas tanto al interior como al exterior de las raíz. La micorriza funciona como órgano de absorción y translocación de agua y nutrientes; es una de las más sobresalientes adaptaciones de la raíz para desenvolverse adecuadamente en el ambiente edáfico (Barea et al. 1984; Le Tacon 1985; Creighoton et al. 1986; Bethlenfalvay, Linderman 1992). El uso de microorganismos como alternativa para mejorar la condición física y la capacidad productiva de los suelos es hoy ya probada a nivel mundial; es por ello, que la inoculación con hongos micorrízicos busca mejorar la capacidad de la planta para explorar un mayor volumen de suelo y con ello mejorar el desarrollo vegetativo y productivo de los cultivos y obtener unas mejores cosechas, lo que además de mejorar el problema edáfico, se convierte en un aporte sustancial adicional para atacar la inseguridad alimentaria que viven las comunidades que habitan estos territorios. El desarrollo de este estudio tuvo como objetivo fundamental evaluar en campo el efecto de la aplicación de cuatro dosis de micorrizas nativas en el desarrollo vegetativo y productivo del maíz chococito cultivado en suelos de aptitud minera en una zona de la cuenca alta del río San Juan, en el departamento del Chocó.

\section{LOCALIZACIÓN DEL ÁREA DE ESTUDIO}

Este trabajo de investigación se realizó en la Estación Ambiental del Alto San Juan (EAASJ), que se encuentra localizada en el corregimiento de Playa de Oro y sus zonas de influencia (Figura 1) a unos 30 minutos por la vía que va desde Tadó a Pereira. La EAASJ, cubre en términos locales el área de influencia del Consejo Comunitario Mayor de Comunidades Negras de ASOCASAN, pertenecientes al municipio de Tadó, departamento del Chocó, Colombia (Plan de Ordenamiento Territorial del Municipio de Tadó, IIAP, 2006).

Posición geográfica. El municipio de Tadó está ubicado en la parte oriental del departamento del Chocó, dentro de la zona alta del río San Juan, con un área aproximada de 878 $\mathrm{km}^{2}$, su cabecera municipal está ubicada a los $76^{\circ} 73^{\prime} 10^{\prime \prime} \mathrm{de}$ longitud occidental, al margen izquierdo del río San Juan y a la derecha del río Manungará (Plan de Desarrollo Municipal de Tadó, 2007-2011).

\section{MÉTODOS}

Esta investigación se llevó a cabo en cuatro fases experimentales:

Fase 1. Reconocimiento del terreno y determinación del área de estudio. La EAASJ se encuentra ubicada en una zona que históricamente se ha utilizado para la explotación minera; se realizó una revisión general de toda el área de influencia y dentro de esta se escogió el sitio para el establecimiento del 


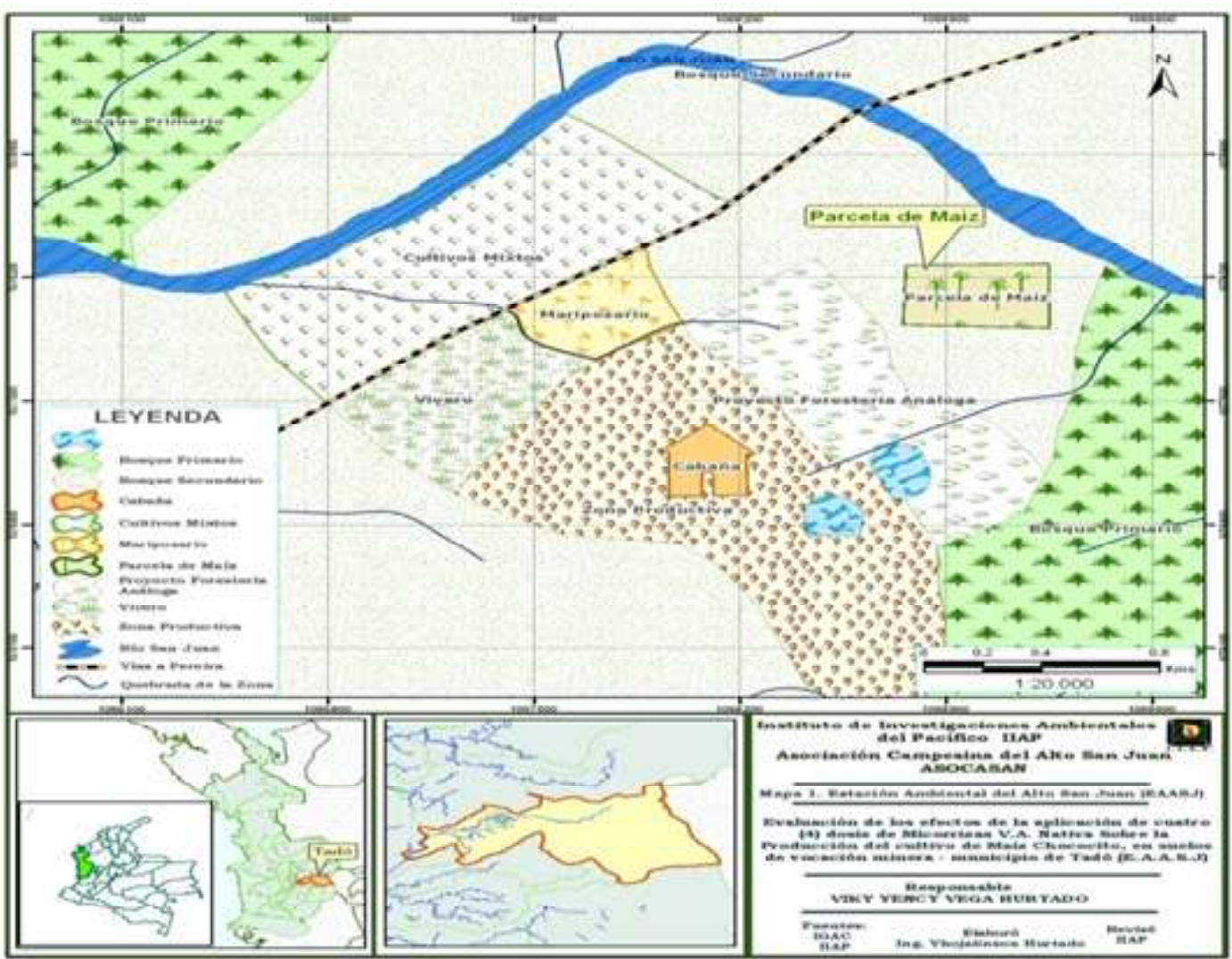

Figura 1. Localización de la Estación Ambiental Alto San Juan

estudio, un área que había sido trabajada con minería y que se encontraba en proceso de recuperación natural, para lo que fue dejada en descanso por más de dos años.

Fase 2. Establecimiento de banco de micorrizas e inoculación de plántulas de maíz chococito. En esta fase se estableció un banco de multiplicación de micorrizas nativas en la estación ambiental de Alto San Juan, a partir de inóculo del género Glomus sp. del banco de germoplasma del Instituto de Investigaciones Ambientales del Pacífico (IIAP) en Tutunendo, que se utilizaron para la inoculación de las plántulas de maíz chococito a nivel de vivero, para su posterior establecimiento en campo. La fertilización del banco de micorrizas se basó en abono orgánico (Figura 2).

Para el establecimiento del banco de multiplicación de la EAASJ, se aplicó el procedimiento metodológico del «Manual de métodos para la investigación de las micorrizas vesículo-arbuscular y otros simbiontes en el laboratorio» de Ewald Sieverding CIAT (1983). La presencia de MVA en la planta se verificó a través del proceso de tinción de raíces, según metodología utilizada por el CIAT.

Para llevar a cabo el proceso de inoculación, se prepararon bolsas de $16 \times 16$, llenadas con un sustrato compuesto por arena lavada, bojeo y hojarasca en proporción $3: 1: 1$; mediante la utilización de una vareta se hizo un orificio en cada una de las bolsas en cuyo fondo se aplicaron las diferentes dosis de micorrizas y se realizó la posterior siembra de las plántulas. El material orgánico se tuvo en cuenta como elemento de mezcla para preparar el sustrato con el que se llenaron las camas de multiplicación de micorrizas y las bolsas de almácigo para la siembra de las plántulas previo trasplante a sitio definitivo.

Fase 3. Establecimiento de parcelas. Se realizó la adecuación del terreno, consecución de materiales e insumos. Se establecieron 12 parcelas totales al azar en bloques, cada una con un área de $6 \mathrm{~m}^{2}$, con una distancia de siembra entre plantas de $1 \mathrm{~m} \times 1 \mathrm{~m}$, para una densidad de 12 plántulas inoculadas por parcela (Figura 3). Se hicieron hoyos de 10 a $20 \mathrm{~cm}$ de profundidad, en los que se depositaron las plántulas con todo el material contenido en las bolsas de almácigo provenientes del vivero. Posteriormente se realizó la siembra de plántulas en sitio definitivo (Figura 4).

Fase 4. Toma de datos, registros y análisis de la información. La recolección de la información de campo se inició una vez sembradas las plántulas de maíz chococito en sitio definitivo, los registros se diligenciaron cada 15 días, se tomaron datos tales como, número de hojas, altura de la planta, diámetro de tallo, número de mazorcas por tratamiento y producción total (Figura 5). 


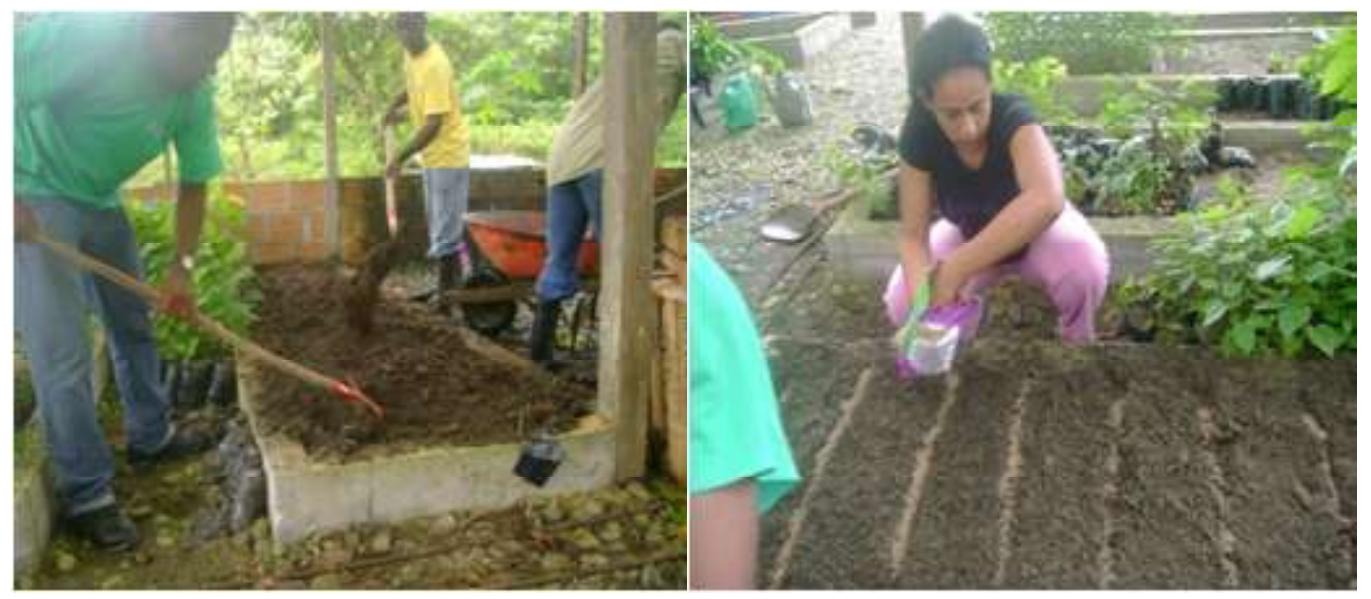

\section{Figura 2. Adecuación de camas de multiplicación y siembra de semilla e inoculación de pasto Brachiaria de cumbens}

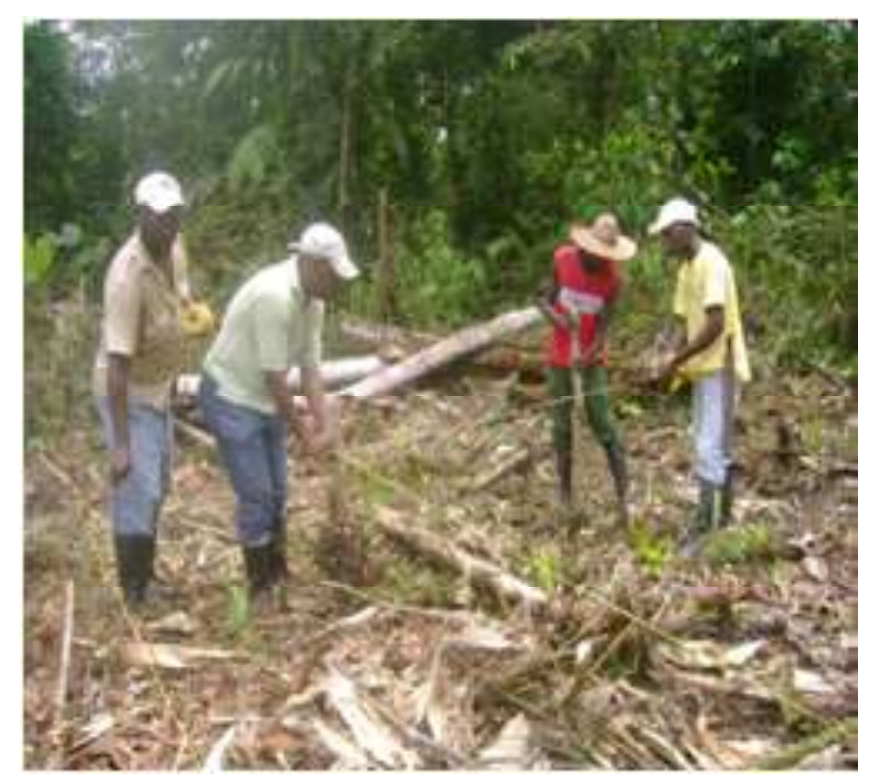

Figura 3. Trazado de unidades experimentales

\section{DISEÑO EXPERIMENTAL}

El ensayo se desarrolló bajo condiciones de campo, con un diseño experimental de bloques completamente al azar. Se evaluaron cepas de hongos micorrízicos: Glomus sp. y el testigo, en un cultivo de maíz chococito. Se establecieron cuatro tratamientos $(0,100,150$ y 250$)$ gramos por planta de inóculo de micorrizas, con tres repeticiones cada uno; para un total de doce unidades experimentales, todas bajo las mismas condiciones agroambientales (temperatura, humedad relativa, precipitación y horas luz/sol).

Las variables evaluadas fueron: área foliar $(\mathrm{cm})$, altura de la planta $(\mathrm{cm})$, diámetro del tallo $(\mathrm{cm})$, número de mazorcas por planta y producción total $(\mathrm{kg})$. La recolección de la información de datos de campo se realizó cada quince días, se elaboraron tablas para la recolección de los datos en campo, para evaluar el efecto de la aplicación de las diferentes dosis de micorrizas-arbusculares sobre unas características o variables medias en plantas de maíz variedad chococito, en la etapa vegetativa y productiva del cultivo. Para ello se aplicó un análisis estadístico o análisis de varianza univariado (ANDEVA), es decir, para cada una de las variables, y el análisis de varianza multivariado.

Las hipótesis propuestas fueron:

Hipótesis 0. La inoculación de plántulas de maíz en etapa de vivero con hongos micorrízicos causa efectos benéficos en el desarrollo vegetativo y productivo de la especie.

Hipótesis 1. La inoculación de plántulas de maíz en etapa de vivero con hongos micorrízicos no causa efectos benéficos en el desarrollo vegetativo y productivo de la especie.

\section{RESULTADOS}

Evaluación de los efectos de la micorriza vesículoarbuscular nativa sobre el desarrollo vegetativo y productivo del cultivo de maíz chococito en suelos con aptitud minera

Efectos sobre el número de hojas. La dosis de $150 \mathrm{~g} /$ planta/ha fue el tratamiento de mejor comportamiento para esta variable, puesto que indujo a la formación de 47 hojas durante todo el ciclo de vida de la planta, seguido de $250 \mathrm{~g} /$ planta/ha con 43 hojas, $100 \mathrm{~g} /$ planta/ha con 36 hojas y finalmente el testigo con 32 hojas.

Los tratamientos 150 y $250 \mathrm{~g} /$ planta generaron diferencias significativas respecto al tratamiento testigo, más no entre ellos. La Figura 6 ilustra el comportamiento de cada uno de los tratamientos evaluados y evidencia el efecto de la efectividad de las dosis aplicadas a plántulas de maíz chococito en etapa de vivero.

Efectos sobre el área foliar $\left(\mathrm{cm}^{2}\right)$. La evaluación de la 


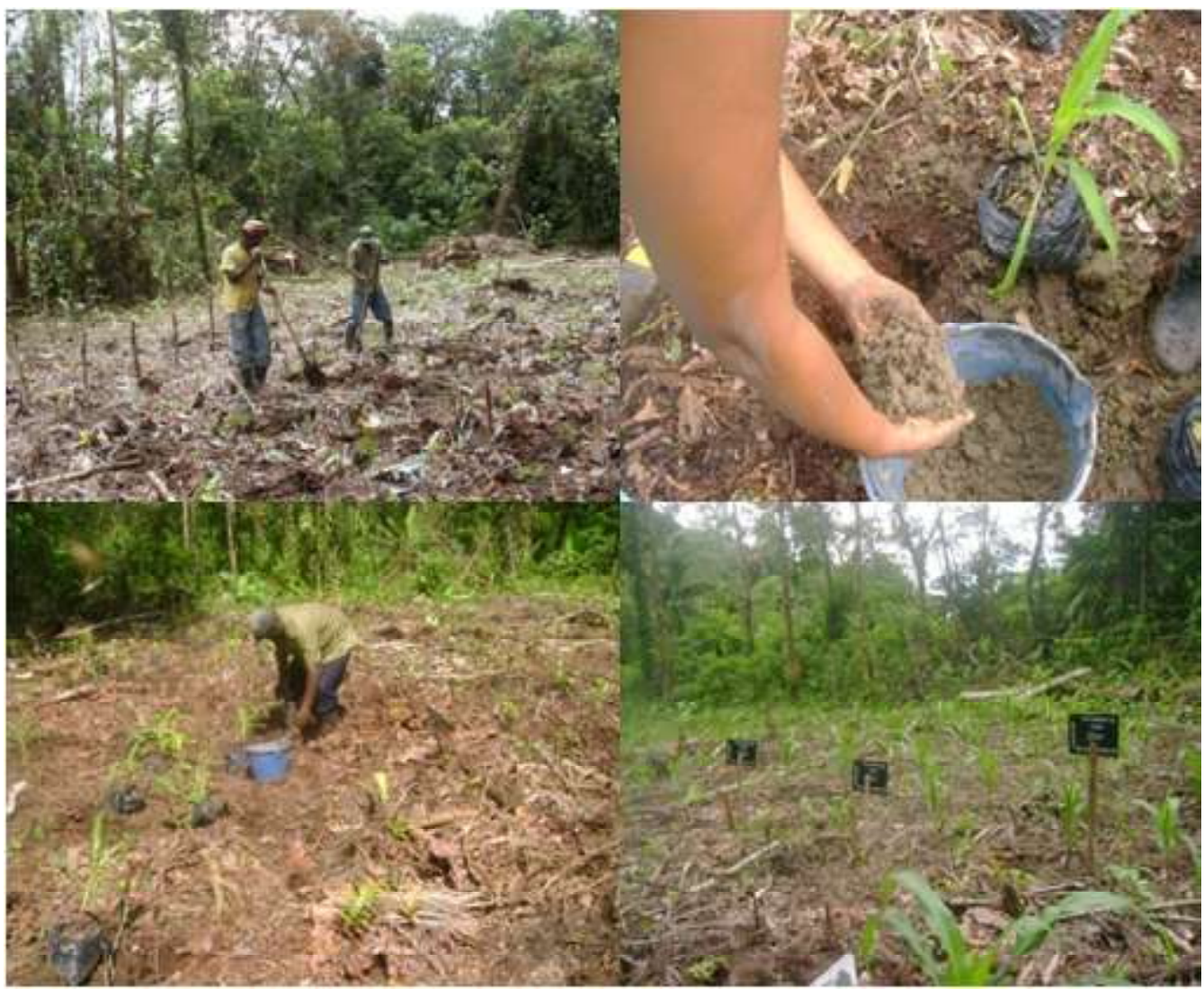

Figura 4. Ahoyado de parcelas y plántulas de maíz inoculadas transplantadas y parcelas establecidas

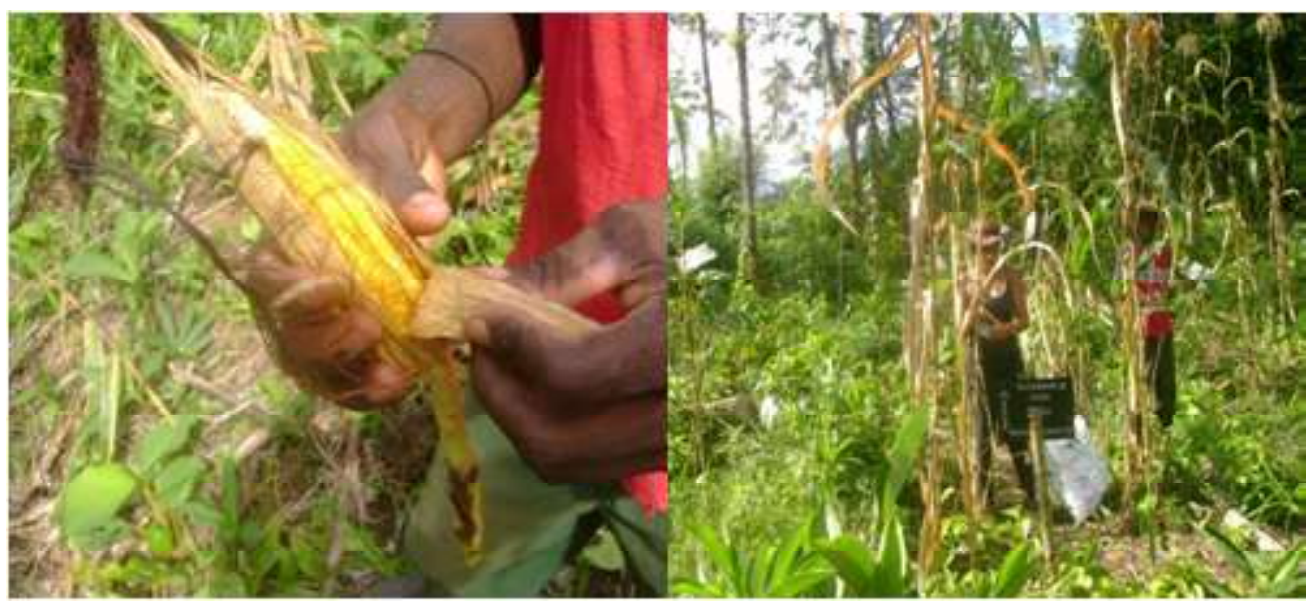

Figura 5. Cosecha de mazorcas y distribución en costales para la toma de datos experimentales

efectividad del inóculo aplicado sobre el área foliar de la hoja de maíz chococito mostró que existe diferencia estadísticamente significativa entre las medias de área foliar de un tratamiento a otro en un nivel de1 $95 \%$ de confianza, es decir, el tipo de tratamiento afectó significativamente a la variable área foliar (la variable área foliar varió según el tratamiento aplicado).
En la Figura 7 se muestra que la dosis $150 \mathrm{~g} /$ planta/ha fue el tratamiento de mayor efectividad porque generó un área foliar media de $3,67 \mathrm{~cm}^{2}$; el tratamiento $250 \mathrm{~g}$ indujo un área foliar de $35,8 \mathrm{~cm}^{2}$, la dosis de $100 \mathrm{~g}$ con $35,2 \mathrm{~cm}^{2}$ y el tratamiento control con $3,35 \mathrm{~cm}^{2}$ de área foliar. Asimismo, se ilustra la relación entre las dosis o tratamientos aplicados y el efecto sobre el área foliar alcanzada por cada uno de ellos, 


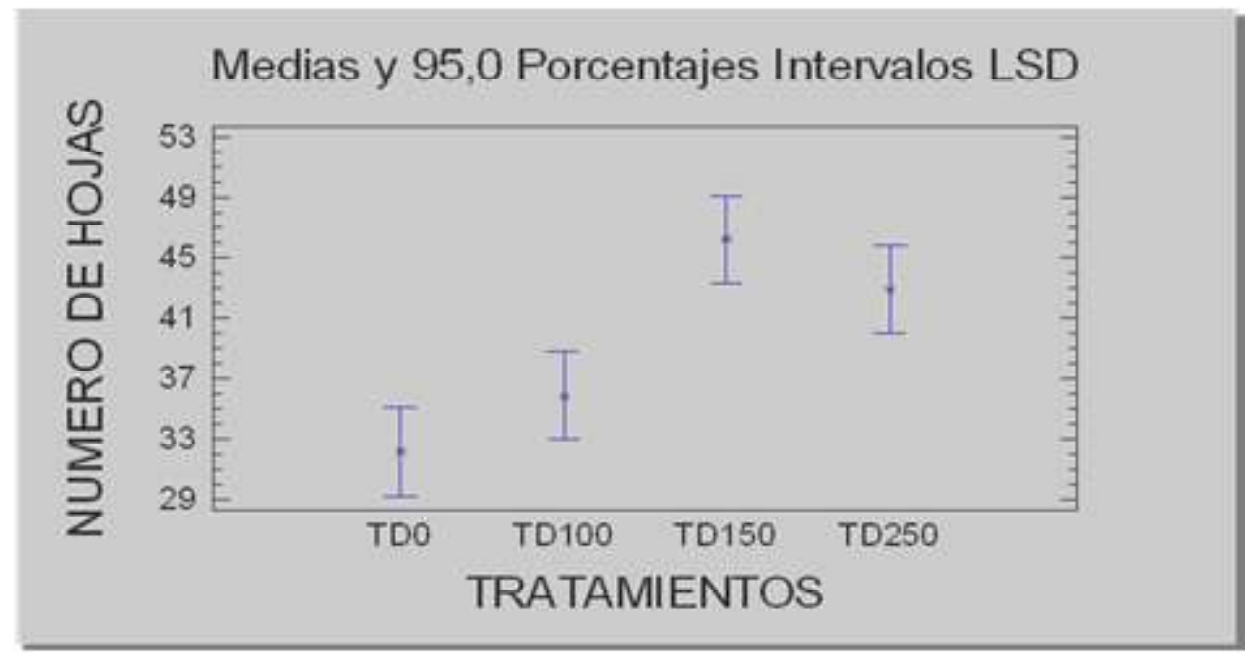

Figura 6. Efectos de dosis de micorrizas VA sobre el número de hojas en maíz chococito

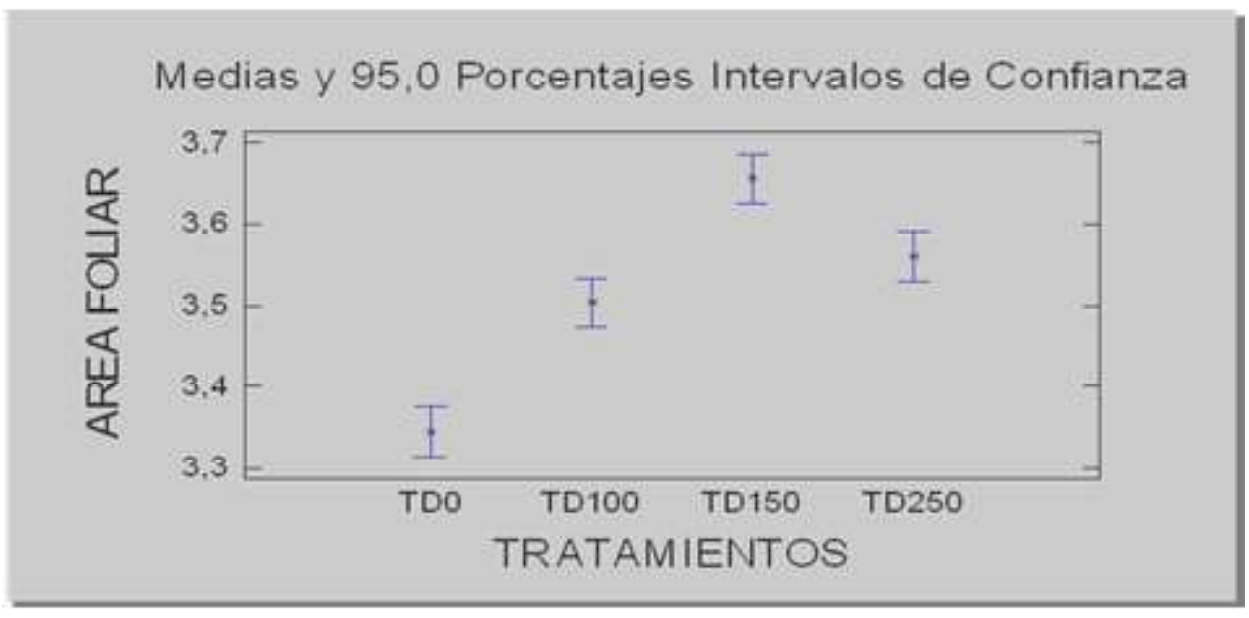

\section{Figura 7. Efectos de la micorriza VA sobre el área foliar en maíz chococito}

respecto al testigo. Todos los tratamientos o dosis de inóculo de micorrizas VA (100 g, $150 \mathrm{~g}$ y $250 \mathrm{~g})$ aplicados a las plántulas de maíz chococito generaron diferencias significativas respecto al tratamiento testigo.

Efectos sobre la altura de la planta (cm). El efecto de la micorriza VA sobre la altura de la planta de maíz chococito, determinó que todos los tratamientos aplicados influyen o afectan significativamente dicha variable. Los resultados muestran que la dosis de $150 \mathrm{~g} /$ planta generó una altura media de $140.849 \mathrm{~cm}$, el tratamiento $250 \mathrm{~g} /$ planta alcanzó $130.020 \mathrm{~cm}$ de altura media, mientras que los tratamientos 100 y 0 g/planta lograron alturas medias de 105.471 y 95.387, respectivamente. Los tratamientos III y IV (150 g y $250 \mathrm{~g})$ mostraron diferencias significativas respecto al tratamiento control.

Efectos sobre el diámetro del tallo (cm). De acuerdo con los resultados alcanzados el inóculo Glomus sp. afecta la variable diámetro del tallo de forma efectiva según la dosis de inóculo aplicado, siendo el tratamiento $150 \mathrm{~g}$, con el que las plantas de maíz lograron el mayor diámetro o grosor del tallo $(\mathrm{M}=1,94661 \mathrm{~cm})$, y la media más baja de diámetro del tallo se alcanza con $\mathrm{TD}_{0}(\mathrm{M}=1,43618 \mathrm{~cm})$.

Las dosis $150 \mathrm{~g}$ y $250 \mathrm{~g}$ lograron la mayor efectividad y diferencia significativa en comparación con el tratamiento control en relación con la variable diámetro o grosor del tallo. En la Figura 8 se comparan y evidencian las diferencias en cuanto a la efectividad de los tratamientos evaluados.

Efectos sobre el número de mazorcas. Se demostró que la inoculación de cepas del genero Glomus sp. influye directamente sobre la variable número de mazorcas. Los resultados alcanzados indican que el número promedio más alto de mazorcas se logró al aplicar $\mathrm{TD}_{250}(\mathrm{M}=3,8)$, generando diferencias significativas con respecto a los otros tratamientos evaluados. Los tratamientos $\mathrm{TD}_{0}, \mathrm{TD}_{100}, \mathrm{TD}_{150}$, no mostraron diferencia significativa entre la media de número de mazorcas producidas con cada una de dichas dosis. La Figura 
9 ilustra el comportamiento de cada uno de los tratamientos evaluados respecto a la variable número de mazorcas.

Efectos sobre el tamaño de la mazorca. Los resultados obtenidos para esta variable indican que no hay diferencias estadísticamente significativas entre las medias de tamaño de mazorca respecto a los tratamientos aplicados, es decir, el tamaño de la mazorca no se afecta por el tipo de tratamiento evaluado.

Efectos sobre el desarrollo radicular. Para la evaluación de esta variable se tuvo en cuenta tanto el ancho como el largo radicular. Los resultados muestran que no hay diferencia estadísticamente significativa entre la media de tratamiento, es decir, el desarrollo radicular no es afectado significativamente por el tipo de tratamiento utilizado.

Efectos sobre la producción final. Se determinó el posible efecto de los tratamientos sobre la producción total, demostrándose que la producción de maíz chococito varía dependiendo del tratamiento empleado. El mayor rendimiento en la producción fue de $1763 \mathrm{~kg} / \mathrm{ha}$ y se alcanzó con una dosis de $150 \mathrm{~g} / \mathrm{planta}$, seguido de $1750 \mathrm{~kg} / \mathrm{ha}$ cuando se aplicaron $250 \mathrm{~g} / \mathrm{ha}$. Los tratamientos $100 \mathrm{y} 0 \mathrm{~g} / \mathrm{planta}$ indujeron una producción de $1027 \mathrm{~kg} / \mathrm{ha}$ y $777,8 \mathrm{~kg} / \mathrm{ha}$ respectivamente, esto corrobora lo dicho por Acevedo (2005), quien sostiene que el desarrollo e índice de área foliar están asociados con la capacidad de la planta para la captación y uso eficiente de la radiación solar en todo su proceso fotosintético, que en definitiva se traduce en un mejoramiento de su productividad y mejores cosechas. Los tratamientos III y IV generaron diferencias significativas en relación con los tratamientos I y II, pero no hay diferencias estadísticamente significativas entre las medias de estos.

\section{CONCLUSIONES}

El análisis de los resultados obtenidos con el desarrollo de esta investigación permite generar las siguientes conclusiones:

Las micorrizas VA del género Glomus sp., inoculadas a plántulas de maíz chococito en su fase de vivero, generan efectos benéficos una vez trasplantadas a campo porque se favorece el desarrollo vegetativo y productivo de esta importante especie agroalimentaria de los pueblos afro e indígenas del Chocó biogeográfico, lo que es muy relevante, pues con ello, se podrá estructurar y fortalecer la implementación de una estrategia de fomento, orientada a garantizar la seguridad alimentaria de estas etnias y garantizar el establecimiento de alternativas para la comercialización de excedentes productivos.

La inoculación de cepas de micorrizas VA nativas, a plántulas de maíz chococito mejoran el proceso de formación y desarrollo foliar, y estimulan a su vez el desarrollo longitudinal y grosor del tallo de las plantas; esta estimulación es de suprema importancia en especies gramíneas (plantas $\mathrm{C}_{4}$ ) como el maíz.

El análisis de la información generada en el proceso de evaluación muestra que la relación simbiótica establecida entre el bioabono inoculado (MVA) y la planta de maíz chococito, fue altamente efectiva y eficaz; esto se evidencia porque todos los tratamientos aplicados generaron incrementos en la productividad por unidad de área respecto al promedio de productividad departamental; es así que la dosis de $150 \mathrm{~g} /$ planta generó un incremento del $235 \%$, seguido de $250 \mathrm{~g} /$ planta con $233,3 \%$, mientras que la dosis de $100 \mathrm{~g} /$ planta aumentó la productividad en un $136,9 \%$.

\section{RECOMENDACIONES}

Adelantar investigaciones que permitan evaluar los efectos de la inoculación con micorrizas nativas con otras especies vegetales agroalimentarias presentes en el Chocó biogeográfico, buscando con ello mejorar condiciones edáficas de los suelos y aumentar niveles de producción, garantizando con ello la dieta alimentaria de las familias rurales de esta importante ecorregión de Colombia.

Identificar y caracterizar las especies nativas de MVA presentes en las áreas de influencia de las estaciones ambientales que el IIAP posee en el Chocó biogeográfico, como estrategia para la multiplicación, fomento y uso de este importante recurso biológico en diferentes acciones productivas y ambientales.

Igualmente, se recomienda establecer estudios relacionados con la evaluación de las propiedades químicas de los sustratos utilizados en esta investigación, porque existe la probabilidad de que estos hayan incidido en la variación de los contenidos iniciales de algunos elementos nutrientes, como por ejemplo, el fósforo (P).

Establecer bancos de germoplasmas, a partir de las especies de MVA nativas colectadas en las estaciones ambientales, evaluando su efectividad y eficacia cuando son inoculadas en diferentes especies agrícolas, forestales, frutales y ornamentales.

\section{LITERATURA CITADA}

Acevedo, H. E. 2005. Fisiología del rendimiento del maiz. Santiago de Chile: Universidad de Chile. 125 pp.

Agudelo, R., N. Martínez, J. E. Ceballos, B. Caicedo, M. Riascos. 2005. Los maíces chococito: base de la autonomía alimentaria y del desarrollo cultural de las comunidades negras en la costa pacífica. Grupo semillas. Experiencias locales del cultivo tradicional del maíz. Colombia. Rev Semillas (22/23): 5-10.

Alcaldía Municipal de Tadó. Plan de Desarrollo Municipal de Tadó 20072011. Tadó: Alcaldía Municipal de Tadó. 258 p.

Barea, J. M., C. Azcón-Aguilar, F. B. Roldán. 1984. Avances recientes en el estudio de la micorriza VA. Formación, funcionamiento y efectos recientes en nutrición vegetal. An Edafol Agrobiol. 43: 659-77.

Creighton, M.J., S. Rajapakse, R. K. Garber. 1986. Vesicular arbuscular 


\section{Bioetnia Volumen 8 No 2 (julio-diciembre), 2011}

mycorrhizal in vegetable crops. Horticult Sci. 21 (4): 974-84.

Dodd, J. C. 1999. Recent advances in understanding the role of arbuscular micorrizas in plant production. In: Soil fertility, soil biology and plant nutrition interrelationships. Lavras: Sociedade Brasileira de Ciência do solo. p. 687-704

Instituto de Investigaciones Ambientales del Pacífico (IIAP). Plan de Ordenamiento Territorial del municipio de Tadó. 2006. Quibdó: Instituto de Investigaciones Ambientales del Pacífico (IIAP).

Ferraris, G. N., L. C. Couretot. 2007. Evaluación de la inoculación con micorrizas en maiz bajo diferentes ambientes de fertilidad. Buenos Aires:AACS-FAUBA. 56 p.

Ferraris, G.N., L. A. Couretot, J. C. Ponsa. 2009. Inoculación con micorrizas en maiz. Buenos Aires: INTA. $10 \mathrm{p}$

Gobernación del Chocó. Secretaría de Agricultura y Medio Ambiente. 2007/ 2008. Evaluaciones Agropecuarias. Quibdó: Gobernación del Chocó. 98 p.

Le Tacon, F. 1985. Las micorrizas, una cooperación entre plantas y hongo. Mundo Cientif. 49 (5): 776-84.

Linderman, R. G. 1994. Role of the VAM fungi in biocontrol. In: F. L. Pfleger, R. G. Lindeman (ed.). Mycorrhizaea and plant health. Minnesota: The American Phytopathological Society. p. 1-17.

Mosquera-Blandón, M. 1994. Estudios de diagnóstico sobre la situación ambiental de la minería en Colombia y sus aspectos técnicos, socioeconómicos y estructurales asociados. Quibdó: Instituto de Investigaciones Ambientales del Pacífico. 123 p.

Martínez, L. B., F. I. Pugnaire. 2009. Interacciones entre las comunidades de hongos formadores de micorrizas arbusculares y de plantas. Algunos ejemplos en los ecosistemas semiáridos. Ecosistemas. 18 (2): 44-54.

Pedersen, C. T., D. M. Sylvia. 1996. Mycorrhiza: ecological implications of plant interations. In: K. G. Mukerji (ed.). Concepts in mycorrhizal research. Holanda: Kluwer Academic Publisher. p. 195-212.

Raman, N., A. Mahadevan. 1996. Mycorrhizal research. A priority in agriculture. In: K. G. Mukerji (ed.). Concepts in mycorrhizal research. Holanda:
Kluiwer Academic Publishers. p. 41-61.

Rodríguez, A. M., I. García, R. Michelena. 2003. Caracterización de la micorrización de plantas de maíz cultivadas en suelo bajo siembra directa. Buenos Aires: Facultad de Agronomía, Universidad de Buenos Aires

Safir, G. R. 1990. Micorrizas arbúsculo-vesicular y la productividad agrícola. En: Biología de la productividad de los cultivos. México, DF: Ed. P. S. Carlson. p. 201-16.

Sieverding, E. 1983. Manual de métodos para la investigación de la micorriza vesículoarbuscular en el laboratorio. Proyecto Micorriza. Palmira: Centro Internacional de Agricultura Tropical (CIAT). p. 209-23.

Sieverding, E. 1984. Importancia de las micorrizas en la nutrición de las plantas. I Curso Nacional de Micorrizas. Palmira: Facultad de Ciencias Agropecuarias, Proyecto Micorrízas, CIAT. p.1-6.

Sieverding, E. 1991. Vesicular arbuscular mycorrhiza management in tropical agrosystems. Alemania: GTZ. 371 p.

Siqueira, J. O. 1985. Importancia e potencial das associações micorrizicas para e agricultura. Belo Horizonte: Empresa de Pesquisa Agropecuaria. $20 \mathrm{pp}$.

Siqueira, J., F. Moreira, A. López, L. Guilherme, V. Faquin, A. Furtini Neto, et al. (Eds.) 1999. Lavras: Sociedade Brasileira de Ciência do Solo. p. 687-704.

Srivastava, D., R. Kapoor, S. K. Srivastava, S. K. G. Mujerki. 1996. Vesicular arbuscular mycorrhiza an overview. In: K. G. Mujerji (ed.). Concepts in mycorrhizal research. Holanda: Kluiwer Academic Publishers. p. 1-24.

Stribley, D.P. 1990. Mycorrhizal associations and their significance. In: Ravinovich, H.D., J.L. Brewster (eds.). Onions and allied crops. II. Agronomy, biotic interactions, pathology and crops protections. Boca Raton: CRC Press. p. 85-101

Uribe, G. 2005. Producción de maiz mediante el uso de biofertilizantes en el sistema roza, tumba y quema en un suelo de tipo cambisol. Yucatán: Instituto Nacional de Investigaciones Forestales Agrícolas y Pecuarias, Centro de Investigación Regional del Sureste. 